\section{Summary of: Type 2 diabetes risk screening in dental practice settings: a pilot study}

\author{
D. Wright, ${ }^{* 1}$ V. Muirhead, ${ }^{2}$ S. Weston-Price ${ }^{3}$ and F. Fortune ${ }^{4}$
}

FULL PAPER DETAILS

${ }^{1}$ Consultant in Dental Public Health, Public Health England, 151 Buckingham Palace Road, London, SW1W 9SZ; ${ }^{2 N I H R ~ A c a d e m i c ~ C l i n i c a l ~ L e c t u r e r / ~}$ Speciality Registrar in Dental Public Health, Centre for Clinical and Diagnostic Oral Sciences, ${ }^{3}$ Clinical Lecturer in Adult Dental Health, ${ }^{4}$ Head of Clinical and Diagnostic Oral Sciences, Institute of Dentistry, Barts and the Royal London School of Medicine and Dentistry, Queen Mary University of London, UK ${ }^{*}$ Correspondence to: Desmond Wright Email:Desmond.wright@phe.gov.uk; Tel: 07768376876

Refereed Paper

Accepted 12 December 2013

DOI: $10.1038 /$ sj.bdj.2014.250

•British Dental Journal 2014; 216: E15

Background Dental surgeries are highlighted in the 2012 NICE guidance Preventing type 2 diabetes: risk identification and interventions for individuals at high risk as a suitable setting in which to encourage people to have a type 2 diabetes risk assessment. Aim To assess the feasibility of implementing a type 2 diabetes risk screening pathway in dental settings using the NICE guidance tool. Method The study was carried out over two weeks in June 2013. The validated tool in the NICE guidance was used to determine risk. This included a questionnaire and BMI measurement used to determine a risk score. Patients were rated low, increased, moderate or high risk. All patients were given written advice on healthy lifestyle. Patients who were moderate or high risk were referred to their general medical practitioners for further investigation. Participating dental teams were asked to nominate a member who would be responsible for overseeing the screening and training the other team members. Results A total of 166 patients took part in the pilot (58\% male, 75\% aged 49 years or younger and 77\% were from BME groups). Twenty-six low risk patients (15.7\%), 61 increased risk patients (36.7\%), 49 moderate-risk patients (29.5\%) and 30 high-risk patients (18.1\%) were identified during the pilot. Fifteen of the 49 patients (30.6\%) identified as moderate-risk and 6 of the 30 high-risk patients (20\%) had visited their GP to discuss their type 2 diabetes risk in response to the screening. Conclusion The pilot suggests that people at risk of developing type 2 diabetes could be identified in primary, community and secondary dental care settings. The main challenges facing dental staff were time constraints, limited manpower and the low number of patients who visited their GP for further advice.

\section{EDITOR'S SUMMARY}

As we know well the general medical practitioner is someone you visit when you feel ill or are worried that you might be, whereas general dental practitioners see patients more frequently for regular checkups. So it's not surprising that dentists have been highlighted by NICE as one group of healthcare professionals who could help to reduce the incidence of diabetes type 2 by carrying out risk screening of their patients.

This seems eminently sensible but it does throw up a few questions: "Are dentists actually happy to do this?' 'Are patients happy to undergo screening in a dental setting?' 'Do dentists have the time to deliver diabetes screening?' 'Do they have the necessary training to do it?'

Regarding dentists willingness to do this, on the whole the answer is a resounding yes. Studies in the US have shown that over three quarters of dentists questioned were willing to conduct screening for diabetes. ${ }^{1}$

A recent study in this journal looked at the attitudes of patients to screening for diabetes in the dental setting and showed that an overwhelming majority of respondents were willing to be screened for medical conditions by a member of the dental team. ${ }^{2}$

So everyone is willing but are they able? In the studies mentioned above, the dentists questioned indicated that despite a general willingness to carry out diabetes screening, the actual practical implementation of screening programmes, time and education could all be sticking points. The authors of this paper used a pilot to assess the feasibility of implementing a type 2 diabetes risk screening pathway in dental settings following NICE guidance. One hundred and sixtysix patients took part in the study which was carried out in two general dental practices, a specialist clinic in a dental hospital and at a dental school outreach clinic over a two-week period.

Dental practice owners taking part in the pilot felt that the screening had had a positive impact on their whole practice and had also equipped their DCPs with new skills. However, screening added 20 minutes to the appointment time and in some cases patients had to wait to be screened if the surgery was busy. The pilot did not calculate the financial impact on practices.

The study shows that the dental team are more than able to identify those at risk of diabetes and recommend a GP visit if advisable. However, such screening will certainly require more time and increased manpower. The authors recommend that for practical implementation to succeed such screening would need to be commissioned as an enhanced service.

The full paper can be accessed from the $B D J$ website (www.bdj.co.uk), under 'Research' in the table of contents for Volume 216 issue 7.

1. Laurence B. Dentists consider medical screening important and are willing to incorporate screening procedures into dental practice. J Evid Based Dent Pract 2012; 12: 32-33.

2. Creanor S, Millward B A, Demaine A et al. Patients' attitudes towards screening for diabetes and other medical conditions in the dental setting. BrDent J 2014; 216: E2.

Ruth Doherty

Managing Editor

DOI: 10.1038/sj.bdj.2014.263 


\section{TO ACCESS THE BDJ WEBSITE TO READ THE FULL PAPER}

- BDA Members should go to www.bda.org.

- Click the 'login' button on the right-hand side and enter your BDA login details.

- Once you have logged in click the 'BDJ' tab to transfer to the BDJ website with full access.

IF YOUR LOGIN DETAILS DO NOT WORK:

- Get a password reminder: go to www.bda.org, click the login button on the right-hand side and then click the forgotten password link.

- Use a recommended browser: we recommend Microsoft Internet Explorer or Mozilla Firefox.

- Ensure that the security settings on your browser are set to recommended levels.

IF YOU HAVE NOT YET SIGNED UP TO USE THE BDA WEBSITE:

- Go to www.bda.org/getstarted for information on how to start using the BDA website.

\section{COMMENTARY}

Over the last 10-15 years, a huge amount of clinical evidence has been published that clearly links periodontitis susceptibility and diabetes. As dental clinicians we are aware of the importance of oral health as part of overall general health, and routinely consider the general health and wellbeing of our patients when we see them. And many (if not most) of our patients will see us (the dental team) on a much more regular basis than they will see their medical doctor. This then provides an opportunity for the dental team to become more engaged with general health screening of patients.

This is the premise behind the research undertaken by Wright and colleagues, in which they implemented a diabetes screening protocol in dental clinical settings (general dental practices, a dental hospital clinic, and a dental school outreach clinic). A diabetes screening tool developed by NICE was utilised for this purpose, and over a two-week period patients over 25 years old who had not received diabetes screening in the previous five years were invited to take part.

What is really interesting about this study is that the great majority of those involved, both patients and dental clinicians, thought that this was a very worthwhile exercise. The dental staff were trained in the use of the diabetes screening tool, and certainly appeared to benefit from, and enjoy, the increased knowledge that this provided them with.

It is important to note that this study was not about dental clinicians diagnosing diabetes, which, of course, would be beyond their scope of practice. However, it does highlight that the dental team can play an important role in identifying patients who are at risk of diabetes, using an already available screening tool. This indicates the importance that the dental team can have in helping to manage the overall health of their patients, who they see on a regular basis.

The main barrier to the screening, as might be expected, was the time required to do it. The clinicians in this research estimated that around 20 minutes was added to the length of each dental appointment in order to carry out the assessment. Clearly, it would not be practical within current contractual arrangements for the typical busy dental practice to allocate this amount of additional time to screening patients for diabetes. Further investment would be necessary, most suitably in the form of additional staff within the practice to carry out the screening. Given the explosion in diabetes prevalence that we are currently witnessing in many countries, including the UK, methods to diagnose diabetes earlier would be important. Indeed, identifying patients who are at risk of developing diabetes would be particularly useful as a preventive strategy, because these individuals could then be helped to implement healthier lifestyles to minimise the risk of developing diabetes.

The authors are to be congratulated for conducting this study, which clearly demonstrates that the dental team is willing and able to be involved in screening for an important systemic disease such as diabetes, and that such screening is welcomed by patients. Undoubtedly, given the close relationship between diabetes and periodontitis, closer working between the medical and dental professions is warranted for the better management of their patients and this study has provided a model which could be evaluated further in large scale clinical trials.

Prof Philip Preshaw, Professor of Periodontology, Newcastle University
IN BRIEF

- Discusses the integration of oral and general health.

- Reports the results of a pilot examining the provision of health screening in dental practices.

- Discusses the extended role of dental care professionals.

- Outlines the challenges involved in delivering screening programmes in dental practices.

\section{AUTHOR QUESTIONS AND ANSWERS}

1. Why did you undertake this research? As dental public health professionals, we feel that dental teams should develop a culture of delivering a holistic service through the integration of oral health and general health. Following the publication of the NICE guidelines to support diabetes prevention programmes, we were keen to explore whether dental professionals could play a part in implementing the guidance. Offering Type 2 diabetes risk screening in dental practice settings provides an important opportunity to potentially identify undiagnosed Type 2 diabetics or people at high-risk who would benefit from healthy eating and lifestyle advice. Our study assessed whether dental teams would be willing to offer Type 2 diabetes risk screening, what impact this could have on busy practice schedules and whether patients would be willing to accept Type 2 diabetes risk screening in dental settings.

\section{What would you like to do next in this} area to follow on from this work? Our ultimate objective is to encourage dental teams to become more involved in screening for general health conditions in dental settings. The nature of the current dental contract would suggest that this would ideally need to be done through a locally enhanced service. In order to develop such a service, we need to better understand the financial impact on practices in delivering this screening pathway and also collect more information of the views of a wide range of dental practice teams and patients. We would therefore like to run the pilot for a longer period, perhaps three to six months, in different general dental practices of varying sizes and locations with varying socio-demographic profiles and carry out a proper economic impact assessment. 